University of Nebraska - Lincoln

DigitalCommons@University of Nebraska - Lincoln

John R. Hardy Papers

Research Papers in Physics and Astronomy

8-2003

\title{
Dielectric Permittivity and Electric Modulus in $\mathrm{Bi}_{2} \mathrm{Ti}_{4} \mathrm{O}_{11}$
}

Jianjun Liu

University of Nebraska at Omaha

Chun-Gang Duan

University of Nebraska at Omaha, cgduan@clpm.ecnu.edu.cn

Wai-Ning Mei

University of Nebraska at Omaha, physmei@unomaha.edu

R. W. Smith

University of Nebraska at Omaha, robertsmith@unomaha.edu

John R. Hardy

University of Nebraska-Lincoln

Follow this and additional works at: https://digitalcommons.unl.edu/physicshardy

Part of the Physics Commons

Liu, Jianjun; Duan, Chun-Gang; Mei, Wai-Ning; Smith, R. W.; and Hardy, John R., "Dielectric Permittivity and Electric Modulus in $\mathrm{Bi}_{2} \mathrm{Ti}_{4} \mathrm{O}_{11}$ " (2003). John R. Hardy Papers. 1.

https://digitalcommons.unl.edu/physicshardy/1

This Article is brought to you for free and open access by the Research Papers in Physics and Astronomy at DigitalCommons@University of Nebraska - Lincoln. It has been accepted for inclusion in John R. Hardy Papers by an authorized administrator of DigitalCommons@University of Nebraska - Lincoln. 


\title{
Dielectric permittivity and electric modulus in $\mathrm{Bi}_{2} \mathrm{Ti}_{4} \mathrm{O}_{11}$
}

\author{
Jianjun Liu ${ }^{\text {a) }}$ \\ Department of Physics, University of Nebraska, Omaha, Nebraska 68182-0266 and Department of Physics \\ and Center for Electro-Optics, University of Nebraska, Lincoln, Nebraska 68588-0111 \\ Chun-Gang Duan, Wei-Guo Yin, and W. N. Mei \\ Department of Physics, University of Nebraska, Omaha, Nebraska 68182-0266 \\ R. W. Smith \\ Department of Chemistry, University of Nebraska, Omaha, Nebraska 68182-0109 \\ J. R. Hardy \\ Department of Physics and Center for Electro-Optics, University of Nebraska, Lincoln, \\ Nebraska 68588-0111
}

(Received 30 January 2003; accepted 7 May 2003)

\begin{abstract}
Frequency and temperature dependences of dielectric permittivity and electric modulus of pure and Ba-doped $\mathrm{Bi}_{2} \mathrm{Ti}_{4} \mathrm{O}_{11}$ were studied in the ranges of $10^{-1}-10^{6} \mathrm{~Hz}$ and $-150-350^{\circ} \mathrm{C}$, respectively. We found that the antiferroelectric phase transition temperature of $\mathrm{Bi}_{2} \mathrm{Ti}_{4} \mathrm{O}_{11}$ decreases with $\mathrm{Ba}$ doping. In the permittivity studies, we also observed dielectric relaxation peaks shift to higher temperature with increasing frequency. Furthermore, in the electric modulus formalism, conducting peaks were uncovered above $150^{\circ} \mathrm{C}$ in addition to the dielectric relaxation peak. We discussed the mechanisms for the dielectric relaxation and conduction processes based on $\mathrm{TiO}_{6}$ octahedra distortion and a space-charge model. (C) 2003 American Institute of Physics.
\end{abstract}

[DOI: $10.1063 / 1.1587685$ ]

\section{INTRODUCTION}

Several compounds exist in the binary system $\left(\mathrm{Bi}_{2} \mathrm{O}_{3}\right)_{m}-\left(\mathrm{TiO}_{2}\right)_{n} \cdot{ }^{1}$ For example, $\mathrm{Bi}_{4} \mathrm{Ti}_{3} \mathrm{O}_{12}(m=2, n=3)$ is a well-known layer-structured ferroelectric with a Curie temperature of $675^{\circ} \mathrm{C}^{2} \mathrm{Bi}_{2} \mathrm{Ti}_{2} \mathrm{O}_{7}(m=1, n=2)$ was also reported to have a ferroelectric phase transition at $700{ }^{\circ} \mathrm{C}$. ${ }^{1} \mathrm{~A}$ diffuse ferroelectric phase transition was observed in $\mathrm{Bi}_{2} \mathrm{Ti}_{3} \mathrm{O}_{9} \quad(m=1, n=3)$ in the temperature range 200-280 ${ }^{\circ} \mathrm{C}^{3}$ However, $\mathrm{Bi}_{2} \mathrm{Ti}_{4} \mathrm{O}_{11}(m=1, n=4)$ was determined to be an antiferroelectric. Subbarao first studied the temperature dependence of the dielectric constants in $\mathrm{Bi}_{2} \mathrm{Ti}_{4} \mathrm{O}_{11}$ at a fixed frequency $\left(10^{5} \mathrm{~Hz}\right)$ and found a phase transition at $250{ }^{\circ} \mathrm{C}^{4}$ Kahlenberg and Bohm investigated the crystal structures of the room temperature phase $\alpha$ and the high temperature phase $\beta .^{5}$ They found that the $\alpha-\beta$ phase transition is antiferroelectric, which is induced by small displacements of the Bi cations parallel and antiparallel alternatively to the $b$ axis in the $\mathrm{TiO}_{6}$ octahedral network, while $\mathrm{Ti}$ and $\mathrm{O}$ atoms almost remain at the same positions before and after the phase transition. The soft mode resulting from the displacements of the Bi atoms was observed by Raman scattering spectra. ${ }^{6}$

Frequency dependence of complex permittivity $\epsilon^{*}$ has been studied for some compounds in the system $\left(\mathrm{Bi}_{2} \mathrm{O}_{3}\right)_{m}-\left(\mathrm{TiO}_{2}\right)_{n}$. A strong low-frequency dielectric dispersion was observed in $\mathrm{Bi}_{2} \mathrm{Ti}_{2} \mathrm{O}_{7}$ (Ref. 1) and $\mathrm{Bi}_{2} \mathrm{Ti}_{3} \mathrm{O}_{9},{ }^{3}$ while $\mathrm{Bi}_{4} \mathrm{Ti}_{3} \mathrm{O}_{12}$ has several dielectric relaxation peaks below $9500^{\circ} \mathrm{C}{ }^{7}$ It is generally accepted that the presence of

${ }^{a)}$ Electronic mail: jliu@unlserve.unl.edu oxygen vacancies in the compounds of the system $\left(\mathrm{Bi}_{2} \mathrm{O}_{3}\right)_{m}-\left(\mathrm{TiO}_{2}\right)_{n}$ plays an important role to the dielectric properties of the polycrystalline ceramics. Jovalekic et al. reported that the accumulation of oxygen vacancies at the surface of grains in $\mathrm{Bi}_{4} \mathrm{Ti}_{3} \mathrm{O}_{12}$ as well as at domain walls within the grains results in a huge increase of the dielectric permittivity due to the appearance of spatial charge polarization. ${ }^{8}$

Since the above results were mostly on ferroelectric compounds in the system $\left(\mathrm{Bi}_{2} \mathrm{O}_{3}\right)_{m}-\left(\mathrm{TiO}_{2}\right)_{n}$, it will be very interesting to study the frequency and temperature dependence of the permittivity of antiferroelectric compounds in this system, such as $\mathrm{Bi}_{2} \mathrm{Ti}_{4} \mathrm{O}_{11}$ (BT). In this article we report the temperature and frequency dependence of the dielectric permittivity and electric modulus in $\mathrm{BT}$ at frequencies $10^{-1}-10^{6} \mathrm{~Hz}$ in the temperature range $-150-350^{\circ} \mathrm{C}$. In order to study the influence of the oxygen vacancies on the dielectric properties of $\mathrm{BT}$, we also investigated a Ba-doped sample $\mathrm{Ba}_{0.02} \mathrm{Bi}_{1.98} \mathrm{Ti}_{4} \mathrm{O}_{11}$ (BBT). We first observed a dielectric relaxation peak in the temperature dependence of the dielectric permittivity and studied its relaxation time distribution. Then, we found two peaks in the temperature dependence of the electric modulus, the peak at lower temperature was attributed to the dielectric relaxation and that at higher temperature was associated with a conduction process. Both processes are thermally activated following the Arrhenius law.

In Sec. II of this article, we briefly describe the experimental method. Then, in Sec. III, we report the experimental results on the dielectric permittivity and electric modulus, and discuss the mechanisms for the dielectric relaxation and 


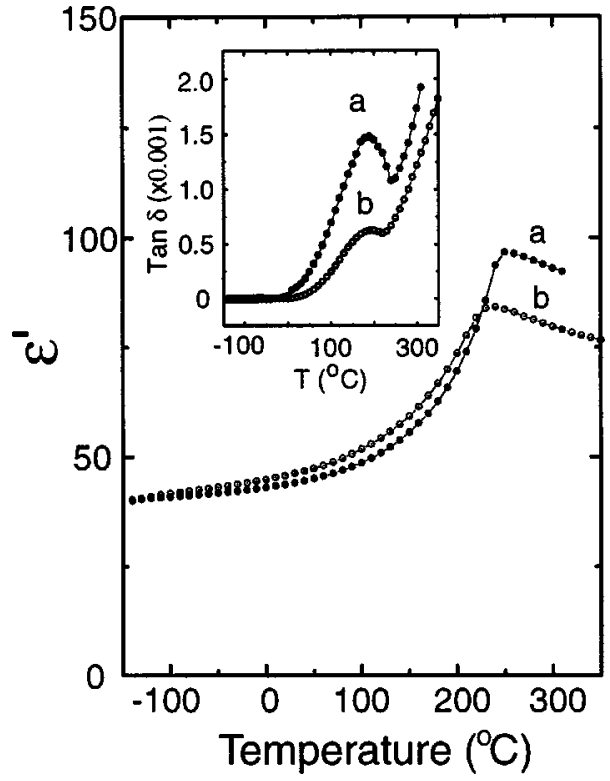

FIG. 1. Temperature dependence of $\epsilon^{\prime}$ and $\tan \delta$ for BT (a) and BBT (b) at the frequency $10^{6} \mathrm{~Hz}$.

the conduction processes. Finally, the conclusion is in Sec. IV.

\section{EXPERIMENT}

The polycrystalline samples $\mathrm{Bi}_{2} \mathrm{Ti}_{4} \mathrm{O}_{11}$ (BT) and $\mathrm{Ba}_{0.02} \mathrm{Bi}_{1.98} \mathrm{Ti}_{4} \mathrm{O}_{11}$ (BBT) were prepared by solid state reaction. The starting materials $\mathrm{Bi}_{2} \mathrm{O}_{3}, \mathrm{TiO}_{2}$, and $\mathrm{BaCO}_{3}$ were dried before being weighed and mixed in an agate mortar thoroughly. The mixtures were calcined in air at 900$1000^{\circ} \mathrm{C}$ for $20 \mathrm{~h}$ with intermediate grinding. The final samples were ground into powder, checked by x-ray diffraction, and pressed into disks of $10 \mathrm{~mm}$ diameter. The disks were sintered at $1100^{\circ} \mathrm{C}$ for $2 \mathrm{~h}$. Complex dielectric permittivities were measured using a NOVOCONTROL Alpha High Resolution Dielectric Analyzer (Alpha-S) in the temperature range $-150-350^{\circ} \mathrm{C}$ and the frequency range $10^{-1}-10^{6} \mathrm{~Hz}$.

\section{RESULTS AND DISCUSSION}

\section{A. Antiferroelectric transition}

The temperature dependence of the dielectric constant (real part of the permittivity) $\epsilon^{\prime}$ and dielectric loss $\tan \delta$ of BT and BBT at frequency $10^{6} \mathrm{~Hz}$ are shown in Fig. 1. $\epsilon^{\prime}$ slightly increases with increasing temperature below $50{ }^{\circ} \mathrm{C}$, then increases rapidly to its maximum at 250 and $240^{\circ} \mathrm{C}$ for $\mathrm{BT}$ and $\mathrm{BBT}$, respectively. After that, $\epsilon^{\prime}$ decreases with increasing temperature. This anomaly is attributed to the antiferroelectric phase transition observed before. ${ }^{5}$ One can see that $\mathrm{Ba}$ doping reduces the phase transition temperature from 250 to $240^{\circ} \mathrm{C}$.

To explain the decrease of the antiferroelectric phase transition temperature by Ba doping, one needs to know the phase transition mechanism in BT. As shown in Fig. 2, the crystal structure of BT consists of double chains of deformed $\mathrm{TiO}_{6}$ octahedra running parallel to the $b$ axis and interlinked

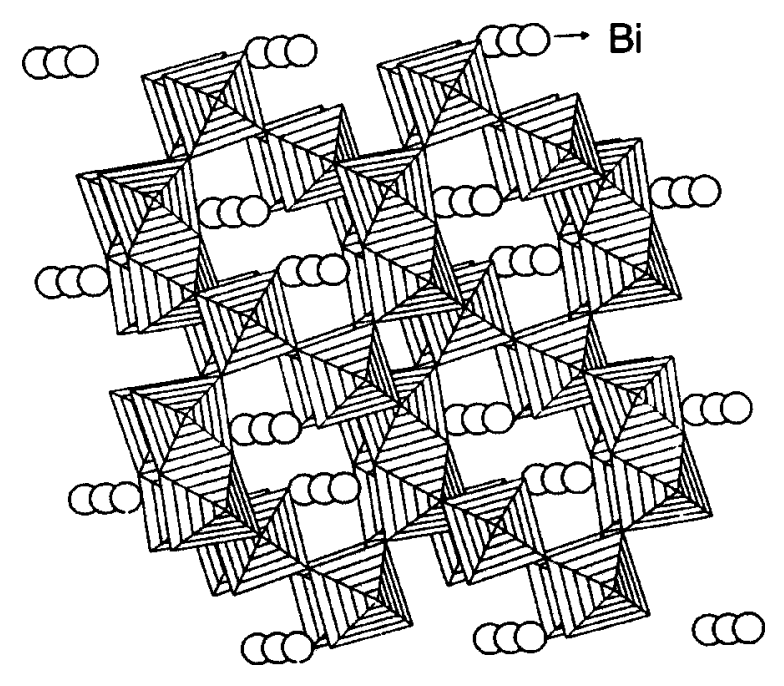

FIG. 2. Projection of the room temperature structure of $\mathrm{Bi}_{2} \mathrm{Ti}_{4} \mathrm{O}_{11}$ deviating $10^{\circ}$ from the $[010]$ direction, the tunnels contain the $\mathrm{Bi}$ cations. (This picture was from Ref. 5.)

partially by edge-sharing and partially by corner-sharing. These octahedra build up a network with channels along the $b$ axis in which the $\mathrm{Bi}^{3+}$ ions are placed. The phase transition is induced by alternating parallel and antiparallel displacements of $\mathrm{Bi}^{3+}$ ions along the $b$ direction resulting in an antiferroelectric polarization of the low temperature $\alpha$ phase. ${ }^{5}$ The displacements of the $\mathrm{Bi}^{3+}$ ions result from the special properties of the $\mathrm{Bi}^{3+}$ ions: i.e., the $\mathrm{Bi}^{3+}$ ions has a lone pair of $6 s$ electrons beyond the closed shell. Hence the possible hybridization of $6 s$ and $6 p$ orbitals makes the $\mathrm{Bi}^{3+}$ ion strongly stereoactive due to the eccentric space occupied by the associated electron cloud. In the high temperature $\beta$ phase, the lone pair electron cloud was proposed to occupy random orientations with respect to the octahedral framework. ${ }^{9}$ Then, on cooling downward, the ordering of the lone pair and the strain of the $\mathrm{TiO}_{6}$ network associated with this ordering induce the antiparallel displacements of the $\mathrm{Bi}^{3+}$ ions along the $b$ axis, and therefore give rise to the lattice distortion. ${ }^{9}$ Thus the replacement of the asymmetric $\mathrm{Bi}^{3+}$ ions by spherically symmetric $\mathrm{Bi}^{2+}$ ions should reduce the distortions, i.e., the $\mathrm{Bi}^{2+}$ ion, which is larger than the $\mathrm{Bi}^{3+}$ ion, symmetric, and less polarizable, tends to occupy the center of the polyhedron and weakens the lattice distortion causing the phase transition temperature to decrease. Hence, in summary, we have demonstrated indirectly that the phase transition in $\mathrm{BT}$ is associated with the displacements of $\mathrm{Bi}^{3+}$ ions along the $b$ axis.

\section{B. Dielectric relaxation}

The temperature dependences of the dielectric permittivity $\epsilon^{*}=\epsilon^{\prime}-i \epsilon^{\prime \prime}$ for various frequencies are shown in Figs. 3 and 4 for $\mathrm{BT}$ and BBT, respectively. At high frequency, above $4.7 \times 10^{5} \mathrm{~Hz}$, we notice that there is a local maximum in $\epsilon^{\prime}$ at about $250^{\circ} \mathrm{C}$, which we believe corresponds to the antiferroelectric phase transition as mentioned in Sec. III A. This local maximum changes to a point of inflection due to 


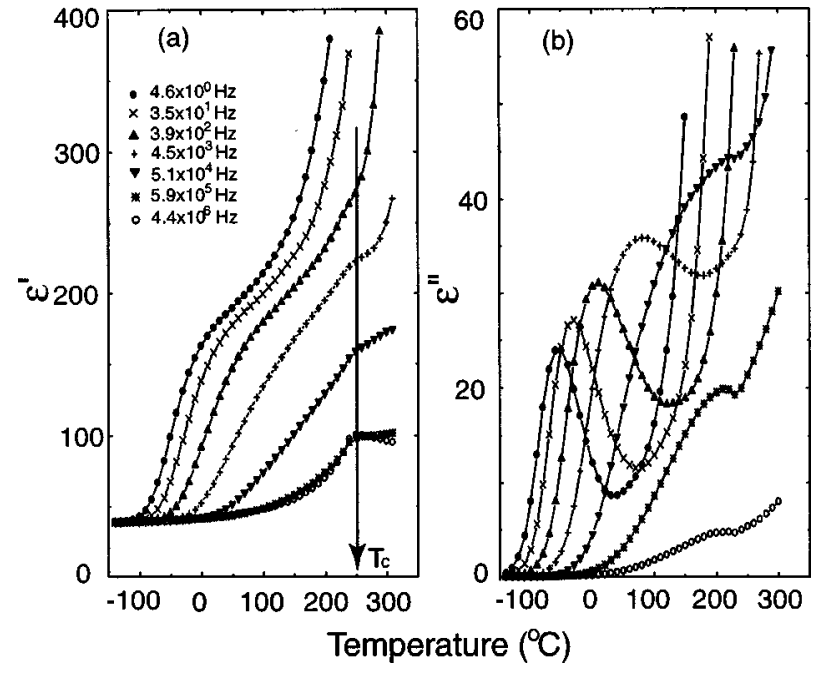

FIG. 3. Temperature dependence of $\epsilon^{\prime}$ (a) and $\epsilon^{\prime \prime}$ (b) for BT at various frequencies.

the rapid increase of $\epsilon^{\prime}$ caused by the electrode surface polarization capacitance, which is much larger than the sample capacitance.

From the imaginary part $\epsilon^{\prime \prime}$ of $\epsilon^{*}$ shown in Fig. 3(b), one can see that at frequencies higher than $5.1 \times 10^{4} \mathrm{~Hz}, \epsilon^{\prime \prime}$ increases monotonically from -150 to $150^{\circ} \mathrm{C}$, then a bump appears at around $200^{\circ} \mathrm{C}$ which shifts to lower temperature as the frequency decreases. This becomes a distinct peak below $4.5 \times 10^{3} \mathrm{~Hz}$, whose position continues to move to lower temperatures at lower frequencies. We attribute this behavior to a low frequency dielectric relaxation. Since the relaxation maximum shifts monotonically to lower temperatures with decreasing frequency, we could ascribe this dielectric dispersion to a thermally activated process. We also notice a rapid increase of $\epsilon^{\prime \prime}$ above the peak, which we believe is due to the conductivity increase of the sample.

In Figs. 5(a) and 5(b), we presented the frequency dependence of $\epsilon^{\prime \prime}$ in BT and BBT, respectively. One can see a relaxation peak shifting to high frequency with increasing

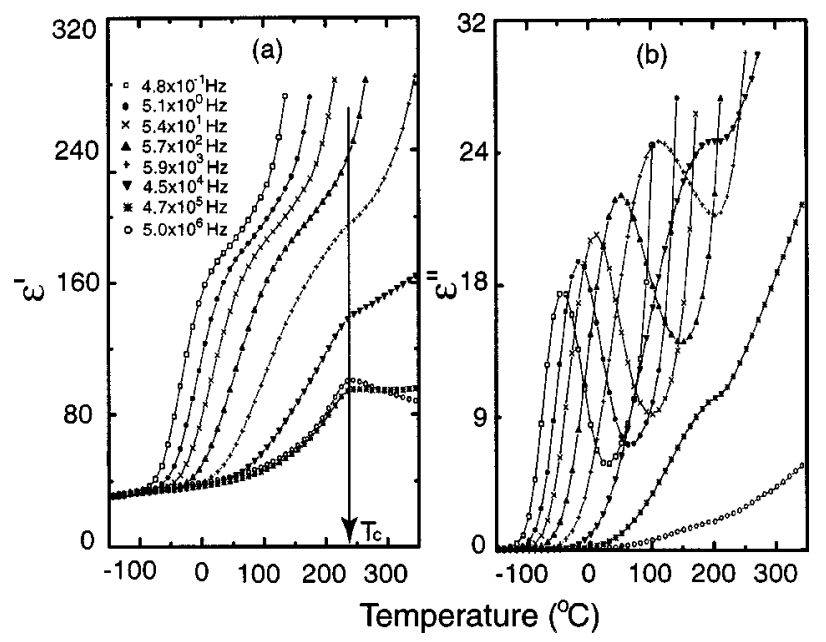

FIG. 4. Temperature dependence of $\epsilon^{\prime}$ (a) and $\epsilon^{\prime \prime}$ (b) for BBT at various frequencies.
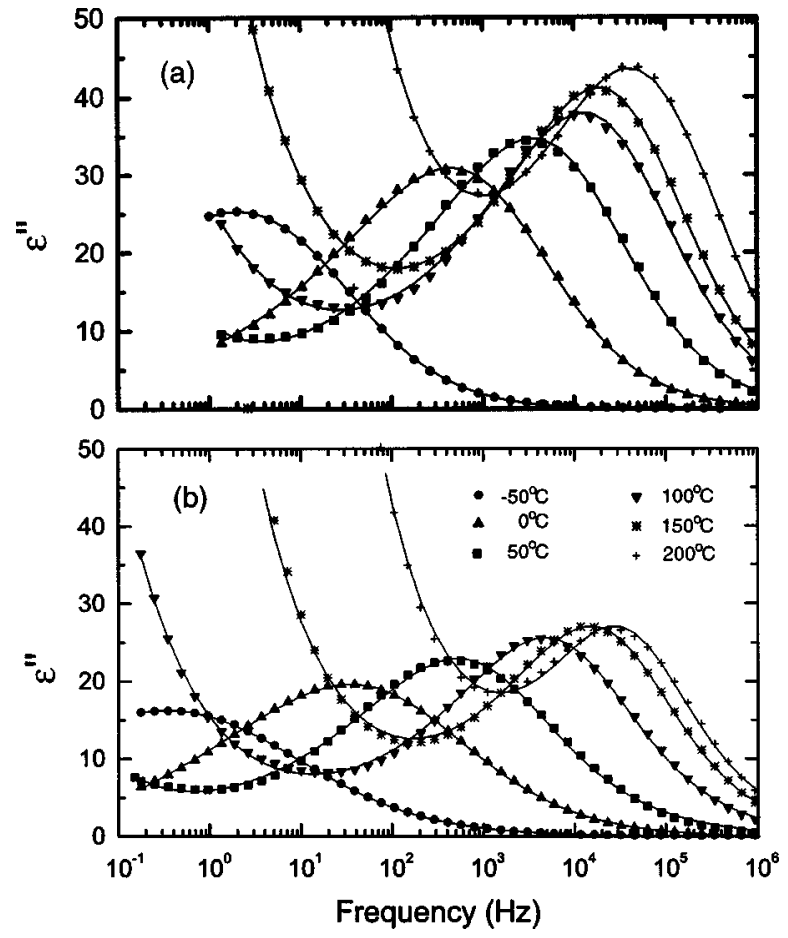

FIG. 5. Frequency dependence of $\epsilon^{\prime \prime}$ for BT (a) and BBT (b) at various temperatures. Solid lines: fitted to the Havriliak-Negami equation (see text). The following fitted parameters are given in the order of temperature $T, \alpha$, and $\beta$. For BT: $\left(-50{ }^{\circ} \mathrm{C}, 0.73,1.98\right) ;\left(0{ }^{\circ} \mathrm{C}, 0.68,1.91\right) ;\left(50^{\circ} \mathrm{C}, 0.65,1.87\right)$; $\left(100^{\circ} \mathrm{C}, 0.62,1.78\right) ;\left(150{ }^{\circ} \mathrm{C}, 0.62,1.75\right)$; and $\left(200^{\circ} \mathrm{C}, 0.67,1.70\right)$. For BBT: $\left(-50^{\circ} \mathrm{C}, 0.76,1.98\right) ;\left(0{ }^{\circ} \mathrm{C}, 0.72,1.78\right) ;\left(50{ }^{\circ} \mathrm{C}, 0.64,1.56\right) ;\left(100{ }^{\circ} \mathrm{C}\right.$, $0.60,1.55) ;\left(150^{\circ} \mathrm{C}, 0.57,1.50\right)$; and $\left(200^{\circ} \mathrm{C}, 0.48,1.30\right)$.

temperature. Meanwhile, on the low frequency side, $\epsilon^{\prime \prime}$ increases rapidly as the temperature increases because of the conductivity contribution mentioned above.

Generally, for a dielectric dispersion with a single relaxation time, the complex permittivity can be described by a Debye equation,

$$
\epsilon^{*}(\omega)=\epsilon^{\prime}(\omega)-i \epsilon^{\prime \prime}(\omega)=\epsilon_{\infty}+\frac{\epsilon_{s}-\epsilon_{\infty}}{1+i \omega \tau}
$$

where $\epsilon_{s}$ and $\epsilon_{\infty}$ are the low- and high-frequency values of $\epsilon^{\prime}, \tau$ is the relaxation time, and $\omega$ is the angular frequency. However, we could not successfully fit the line shape in Fig. 5 by using Eq. (1). Hence we change to the empirical Havriliak-Negami equation, ${ }^{10}$ which assumes a relaxation time distribution,

$$
\epsilon^{*}(\omega)=\epsilon^{\prime}(\omega)-i \epsilon^{\prime \prime}(\omega)=\epsilon_{\infty}+\frac{\epsilon_{s}-\epsilon_{\infty}}{\left[1+(i \omega \tau)^{(1-\alpha)}\right]^{\beta}},
$$

where the parameters $\alpha$ and $\beta$ are used to measure the departure from the ideal Debye response for which $\alpha=0, \beta$ $=1$, and $\tau$ is the mean relaxation time. We fitted the low temperature $\epsilon^{\prime \prime}$ (below $50^{\circ} \mathrm{C}$ ) to Eq. (2) with the constraints $0 \leqslant \alpha \leqslant 1,(1-\alpha) \beta \leqslant 1$. At high temperature (above $50^{\circ} \mathrm{C}$ ) $\epsilon^{\prime \prime}$ was fitted using Eq. (2) together with a conductivity contribution $-i \sigma / \omega^{s}$, where $\sigma$ is the dc conductivity and $s$ is the exponent. In Fig. 5 we showed the fitted results, where the parameter $\alpha$ is in the range $0.5-0.8$ and $\beta$ lies between 1 and 

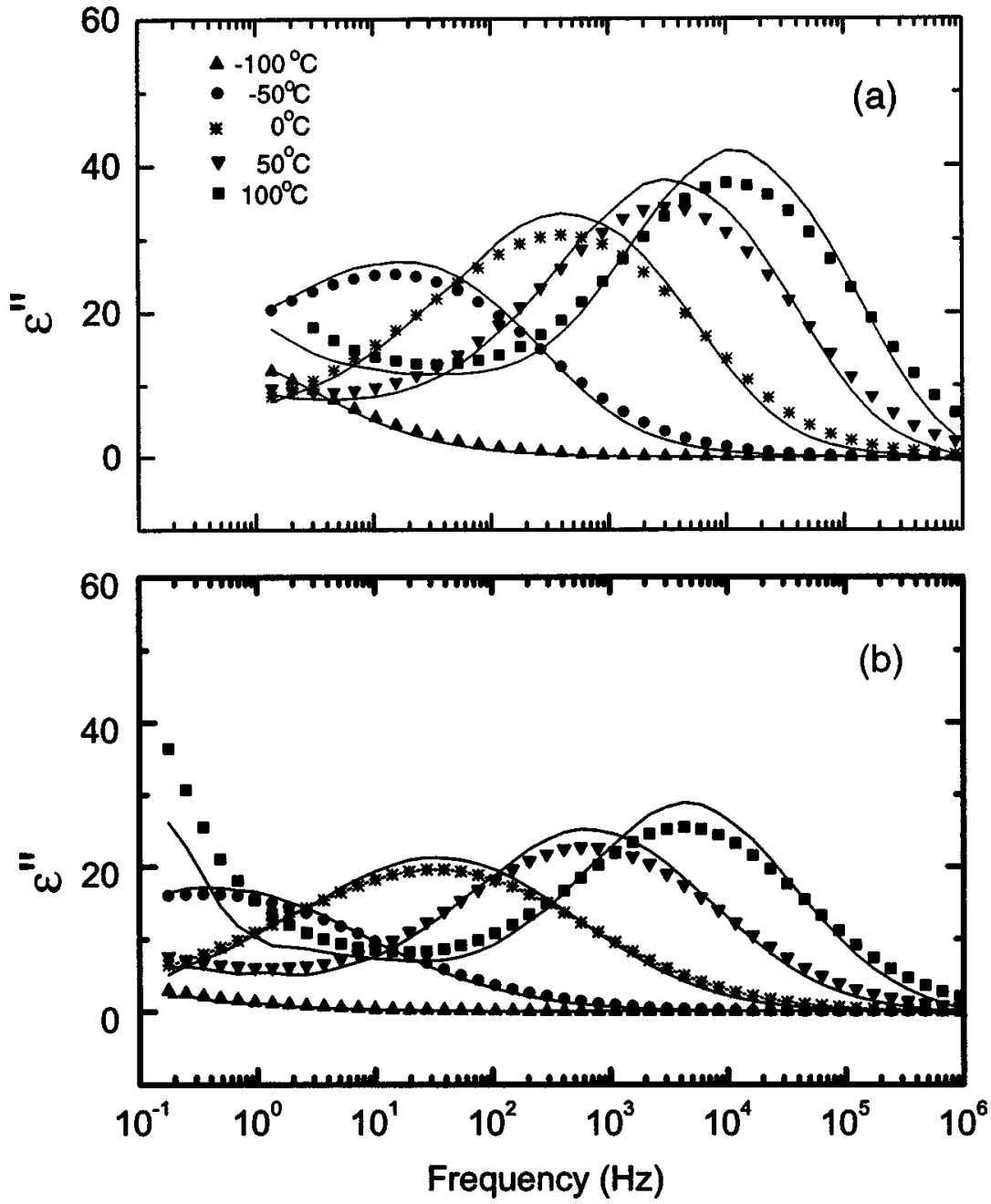

FIG. 6. Comparison of the measured $\epsilon^{\prime \prime}$ with the calculated $\epsilon^{\prime \prime}$ from Eq. (6) at several different temperatures for BT (a) and BBT (b), where the solid lines represent the calculated results.
2. These parameters manifest that the dielectric dispersion in $\mathrm{BT}$ and BBT has a wide relaxation time distribution.

The above-mentioned broad relaxation time distribution can be studied from another angle: ${ }^{11}$ Let $g(\tau, T)$ be the temperature-dependent distribution function for relaxation times, then the complex dielectric constant can be expressed as a superposition of Debye relaxations with different characteristic times $\tau$ (Ref. 12)

$$
\epsilon^{*}-\epsilon_{\infty}=\epsilon(0, T) \int \frac{g(\tau, T) d(\ln \tau)}{1-i \omega \tau},
$$

where $\epsilon(0, T)$ is the low-frequency dielectric constant. Thus the imaginary part of $\epsilon^{*}$ can be written as

$$
\epsilon^{\prime \prime}=\epsilon(0, T) \int \frac{g(\tau, T) d(\omega \tau)}{1+\omega^{2} \tau^{2}} .
$$

For a broad relaxation time distribution function $g(\tau, T)$, when $\tau_{\min } \leqslant 1 / \omega \leqslant \tau_{\max }, \epsilon^{\prime \prime}(\omega, T)$ can be approximated as ${ }^{13}$

$$
\epsilon^{\prime \prime}(\omega, T) \simeq \frac{\pi}{2} \epsilon(0, T) g\left(\frac{1}{\omega}, T\right) .
$$

Hence the spectrum of dielectric relaxation gives direct information about $g(1 / \omega, T)$. In the limit of a broad spec- trum, one can also obtain an important simple relation between the real and imaginary parts of the dielectric permittivity $^{14}$

$$
\epsilon^{\prime \prime}(\omega, T) \simeq-\frac{\pi}{2} \frac{\partial \epsilon^{\prime}(\omega, T)}{\partial(\ln \omega)} .
$$

Thus adopting Eq. (6) we could calculate $\epsilon^{\prime \prime}(\omega, T)$ by using $\epsilon^{\prime}(\omega, T)$ measured at different temperatures. In Fig. 6 we showed the measured values of $\epsilon^{\prime \prime}(\omega, T)$ and those calculated by using the above method. One can see that they show good agreement which verifies the early assumption that, in the measured temperature range, the relaxation time distribution $g(1 / \omega, T)$ is rather broad.

One more important issue worth emphasizing is that based on Eq. (5), apart from the frequency independent prefactor $(\pi / 2) \epsilon(0, T), \epsilon^{\prime \prime}(\omega, T)$ reflects directly the spectral function $g(1 / \omega, T)$. Thus if we plot the $\epsilon^{\prime \prime}(\omega, T)$ data in scaled coordinates, i.e., $\epsilon^{\prime \prime}(\omega, T) / \epsilon_{\max }^{\prime \prime}$ versus $\log \left(\omega / \omega_{m}\right)$, where $\omega_{m}$ corresponds to the frequency of the relaxation peak in the $\epsilon^{\prime \prime}$ versus $\log (\omega)$ curve, we can deduce the shape of the relaxation time spectrum $g(1 / \omega, T)$ at different temperatures, as shown in Fig. 7. In view of the slight spread of the curves, we conclude that the distribution function for 

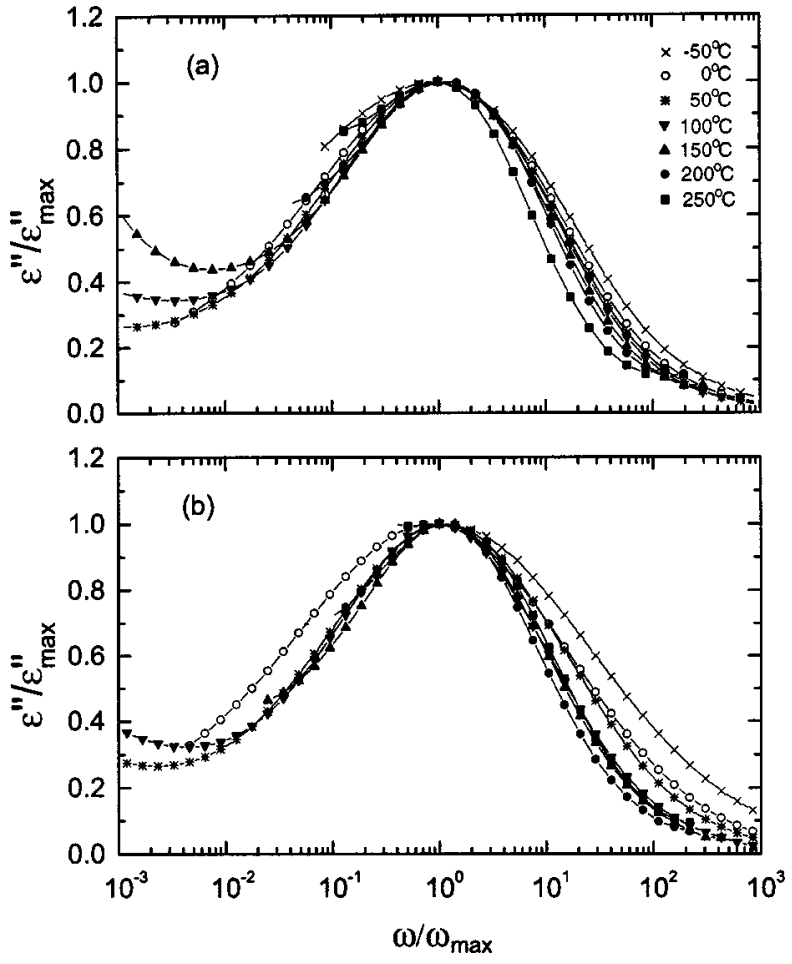

FIG. 7. Scaling behavior of $\epsilon^{\prime \prime}$ at various temperature for BT (a) and BBT (b).

relaxation times has a mild temperature dependence, its width decreasing with increasing temperature.

After we obtain the relaxation time distribution $g(\tau, T)$, we could also determine the most probable relaxation time $\tau_{m}=1 / \omega_{m}$ from the position of the relaxation peak in the $\epsilon^{\prime \prime}(\omega, T)$ versus $\log \omega$ plots. This generally follows the Arrhenius law:

$$
\tau_{m}=\tau_{o} \exp \left(E_{a} / k_{B} T\right),
$$

where $\tau_{o}$ is the prefactor and $E_{a}$ denotes the activation energy for dielectric relaxation. The Arrhenius law was originally developed to model dielectric properties of gases and polar liquids, and assumes a temperature independent activation energy. In Fig. 8 we plot the measured values of $\ln \tau$ versus $1 / T$, and the solid lines are the fitted results using Eq. (7). From the slopes of these straight lines we obtain activation energies of 0.33 and $0.41 \mathrm{eV}$ for BT and BBT, respectively. We see that Ba doping increases the activation energy, a fact that will be discussed in Sec. III D.

\section{Electric modulus}

When discussing the results presented in Fig. 5, we noted that $\epsilon^{\prime \prime}$ increases with temperature in the low frequency region, which we attributed to a conduction process. In order to explore this phenomenon in detail, we use the electric modulus formalism $M^{*}$ for rendering.

The electric modulus is the reciprocal of the permittivity $M^{*}=1 / \epsilon^{*}$. Although it was originally introduced by Macedo ${ }^{15}$ to study space charge relaxation phenomena, $M^{*}$ representation is now widely used to analyze ionic conductivities. ${ }^{16}$ Generally, for a pure conduction process, a relaxation peak would be observed in the frequency spectra

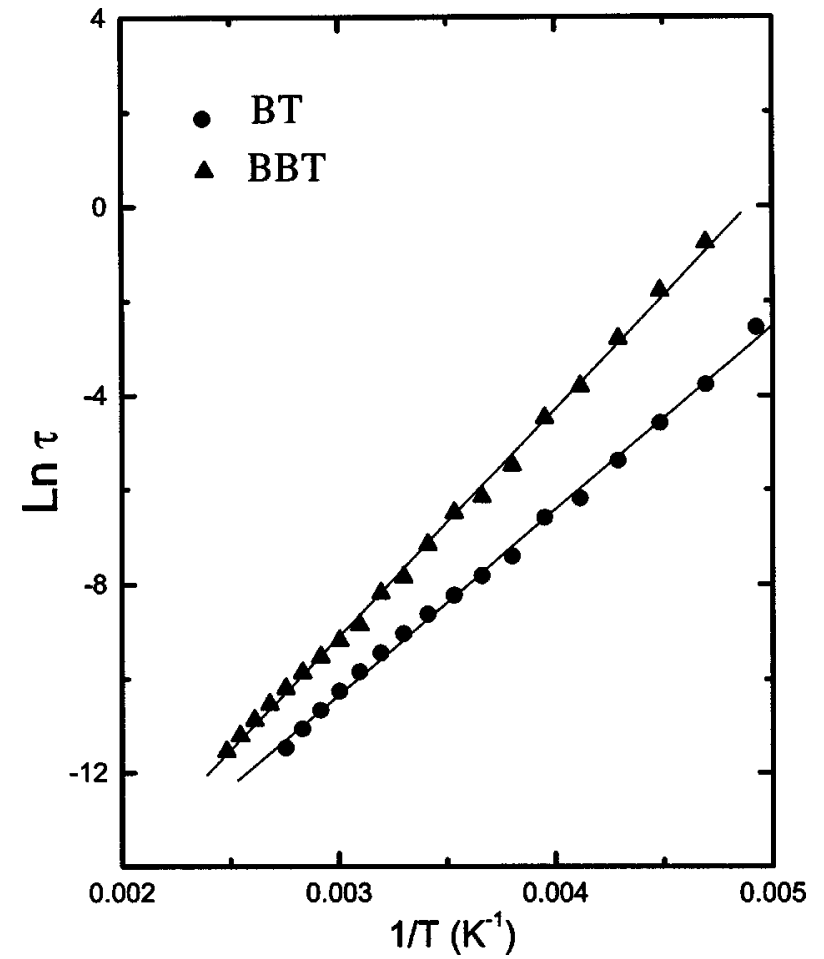

FIG. 8. Arrhenius plot of dielectric relaxation times for BT and BBT.

of the imaginary component $M^{\prime \prime}$ and no peak would take place in the corresponding plot of $\epsilon^{\prime \prime}$. However, for a dielectric relaxation process, a relaxation peak appears in both the $M^{*}$ and $\epsilon^{*}$ representation. Comparisons of the $\epsilon^{*}$ and $M^{*}$ representations have been used to distinguish localized dielectric relaxation processes from long-range conductivity. ${ }^{17,18}$

Physically, the electric modulus corresponds to the relaxation of the electric field in the material when the electric displacement remains constant, so that the electric modulus represents the real dielectric relaxation process, which can be expressed as ${ }^{15}$

$$
\begin{aligned}
M^{*}(\omega)=1 / \epsilon^{*}(\omega) & =M^{\prime}+i M^{\prime \prime} \\
& =M_{\infty}\left[1-\int_{0}^{\infty}\left(-\frac{d \Phi(t)}{d t}\right) \exp (-i \omega t) d t\right]
\end{aligned}
$$

where $M_{\infty}=\left(\epsilon_{\infty}\right)^{-1}$ is the asymptotic value of $M^{\prime}(\omega)$, and $\Phi(t)$ is the time evolution of the electric field within the material.

The temperature dependence of the electric modulus $M^{\prime}$ and $M^{\prime \prime}$ for various frequencies is shown in Figs. 9 and 10 for BT and BBT, respectively. Both samples show a two-step decrease in $M^{\prime}$ in the measured temperature range. One is in the range of -150 to $100{ }^{\circ} \mathrm{C}$ and the other lies between 100 and $350^{\circ} \mathrm{C}$. Correspondingly, $M^{\prime \prime}$ shows two peaks in the same temperature ranges, both peaks shift to high temperature with increasing frequency. We attribute the low temperature peak to the dielectric relaxation mentioned in Sec. III B, and the high-temperature peak to the conduction process. Furthermore, we notice that the conduction peak in BBT 


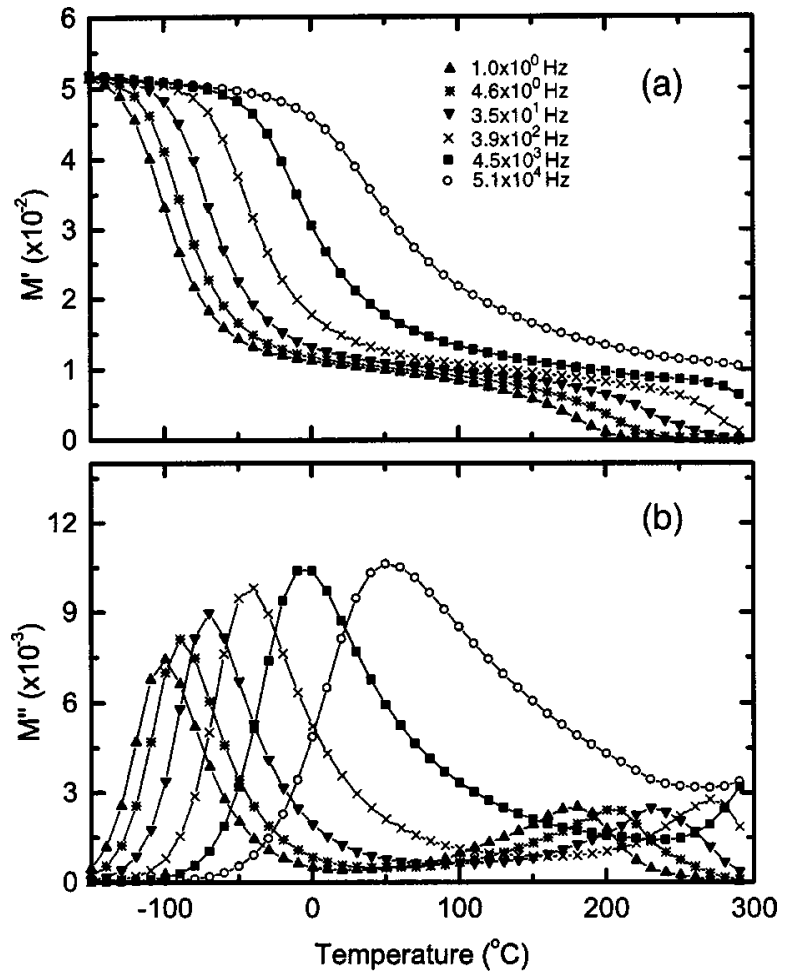

FIG. 9. Temperature dependence of $M^{\prime}$ (a) and $M^{\prime \prime}$ (b) for BT at various frequencies.

appears at a lower temperature than that of $\mathrm{BT}$, which means Ba doping decreases the activation energy of the conduction process.

In Fig. 11 we show the frequency dependence of $M^{\prime \prime}$ at various temperatures. We observe that two peaks appear in $M^{\prime \prime}$ above $150^{\circ} \mathrm{C}$, which shift to higher frequency with increasing temperature. These peaks indicate the transition from short range to long range mobility with decreasing frequency, where the low frequency side of the peak represents the range of frequencies in which the ions are capable of moving long distances, i.e., performing successful hopping from one site to the neighboring site, whereas, for the high frequency side, the ions are spatially confined to their potential wells and can execute only localized motion. ${ }^{19}$

We derive the most probable conductivity relaxation times $\tau_{c}$ from the peak frequency $f_{\max }$ in $M^{\prime \prime}$ based on the condition $2 \pi f_{\max } \tau_{c}=1$, then plot their reciprocal temperature dependencies in Fig. 12. We observe that they follow closely the Arrhenius law [Eq. (7)]. Based on the high temperature data, we obtained the activation energies for conduction processes, 1.63 and $1.24 \mathrm{eV}$ for BT and BBT, respectively, while using both high and low temperature data we deduced activation energies for dielectric relaxation of 0.33 and $0.41 \mathrm{eV}$, respectively, which are consistent with the values obtained in Sec. III B. We can clearly see that Ba doping increases the activation energy of the dielectric relaxation, but decreases that of the conduction process.

\section{Mechanism for dielectric relaxation and conduction}

From studying the dielectric response, we found both dielectric relaxation and a conduction process in BT and
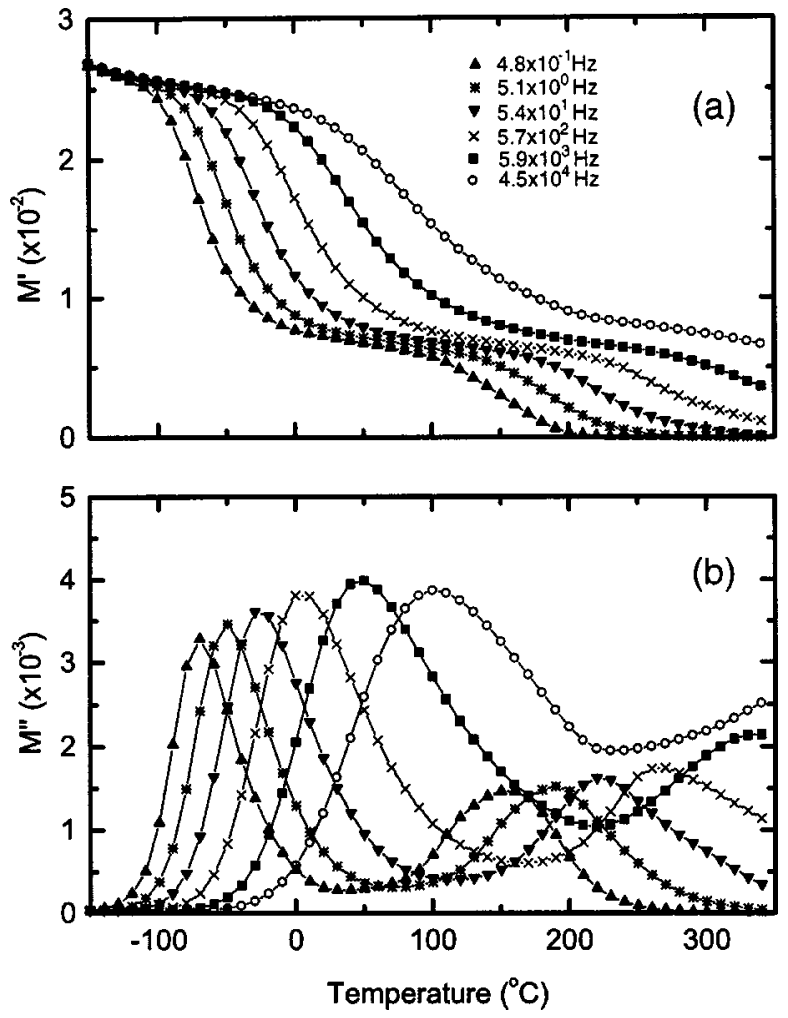

FIG. 10. Temperature dependence of $M^{\prime}$ (a) and $M^{\prime \prime}$ (b) for BTT at various frequencies.

BBT. As shown in Sec. III B, the dielectric relaxation has several characteristics: first, it has a broad relaxation time distribution with the width decreasing with increasing temperature. Second, it is a thermally activated process following the Arrhenius law with an activation energy of $0.33 \mathrm{eV}$ for BT and $0.41 \mathrm{eV}$ for BBT. Third, this process persists above the antiferroelectric phase transition temperature $T_{c}$. All the aforementioned behaviors could be explained by the commonly used Skanavi model. ${ }^{20,21}$ To account for the origin of a dielectric relaxation observed in $\mathrm{Bi}_{2} \mathrm{O}_{3}$-doped $\mathrm{SiTiO}_{3}$, Skanavi suggested that the distortions of the oxygen octahedra that surround the $\mathrm{Ti}$ ions introduced by $\mathrm{Bi}^{3+}$ ions and associated vacancies are sufficient to produce more than one off-center equilibrium position for the $\mathrm{Ti}^{4+}$ ion, and that the observed relaxation is associated with thermally activated motion among these equivalent minima.

The structure of $\mathrm{Bi}_{2} \mathrm{Ti}_{4} \mathrm{O}_{11}$ is formed by a framework of distorted $\mathrm{TiO}_{6}$ octahedra, i.e., in the low temperature phase, the individual Ti-O distance varies from 1.797 to $2.200 \AA$, and the angles between the central atoms and the corners of the octahedra show a pronounced deviation from the ideal values $90^{\circ}$ and $180^{\circ} .5$ Thus, following the reasoning of the Skanavi model, ${ }^{20,21}$ one can assume that several potential minima for Ti ions may exist in the distorted oxygen octahedra. Therefore the dielectric relaxation described in Sec. III B could be associated with thermally activated motion of $\mathrm{Ti}$ ions hopping among them. Since these distortions persist above the antiferroelectric phase transition, ${ }^{5}$ one expects to observe the dielectric relaxation above $T_{c}$ as well. Furthermore, there are two crystallographic inequivalent symmetry positions $\operatorname{Ti}(1)$ and $\operatorname{Ti}(2)$ in the crystal lattice, which are co- 

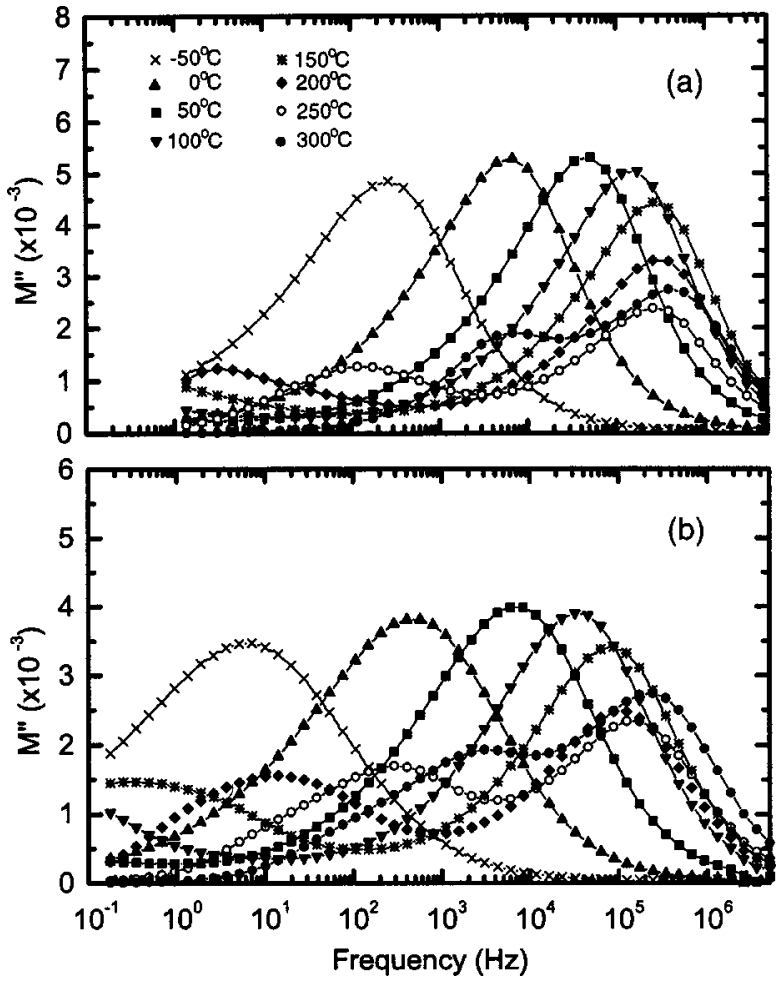

FIG. 11. Frequency dependence of $M^{\prime \prime}$ for BT (a) and BBT (b) at various temperatures.

ordinated by octahedra with different distortions, ${ }^{5}$ and in each of them, different potential minima for the Ti ions will have different strengths. Hence the hopping of Ti ions among these minima results in a broad relaxation time distribution. In addition, at high temperature, the more thermally excited Ti ions, which carry higher kinetic energy than some potential wells, hop less discriminately among all these potential minima, thus reducing the relaxation time distribution.

As for the activation energy increase from $0.33 \mathrm{eV}$ in BT to $0.41 \mathrm{eV}$ in BBT, we attribute that to additional distortion of the oxygen octahedra caused by oxygen vacancies introduced by replacing $\mathrm{Bi}^{3+}$ ions by $\mathrm{Ba}^{2+}$ ions. The electrical imbalance caused by the divalent $\mathrm{Ba}$ ion substituting for the trivalent $\mathrm{Bi}$ ions is compensated for by the creation of oxygen vacancies, i.e., two doped $\mathrm{Ba}^{2+}$ ions substitute for two $\mathrm{Bi}^{3+}$ ions with the formation of an extra oxygen vacancy. Thus the oxygen vacancies created by Ba doping could further enhance the distortion of oxygen octahedra, and lead to the increase of the activation energy. ${ }^{22}$ This explanation does not conflict with the mechanism of the antiferroelectric phase transition mentioned in Sec. III A because the phase transition is associated only with the alternating $\mathrm{Bi}^{3+}$ ion displacements rather than the distortions of octahedra.

It was reported by $\mathrm{Waser}^{23}$ that the oxygen vacancies can move at higher temperature in $\mathrm{SrTiO}_{3}$ due to thermal activation, with energy of $1.005-1.093 \mathrm{eV}$, and give rise to conduction. Whereas, the conduction activation energies observed in our samples, which are $1.63 \mathrm{eV}$ in $\mathrm{BT}$ and $1.24 \mathrm{eV}$ in BBT, appeared to be too high to attribute to the aforementioned mechanism. The other alternative is the space-charge model, according to Coelho et al. ${ }^{24}$ the free charges move

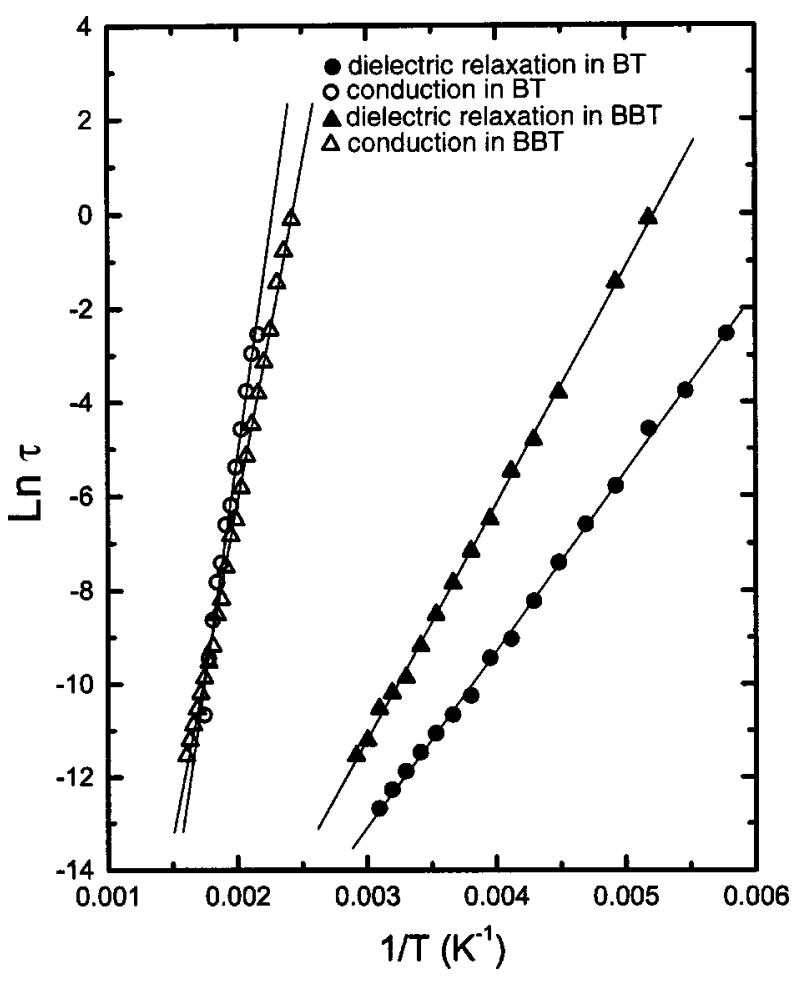

FIG. 12. Arrhenius plot of conduction relaxation times for BT and BBT.

through the sample towards the electrode of opposite sign in the presence of the external electric field and constitute a macrodipole. Therefore its oscillations with external ac field give rise to relaxation and conduction processes. Maglione et $a .^{25}$ explained dielectric relaxation phenomena in several perovskite materials based on this model. They found the relaxation activation energies of space charge were around $1.17-1.48 \mathrm{eV}$ and the conduction activation energies were in the range of $1.07-1.31 \mathrm{eV}$ for La-doped $\mathrm{PbTiO}_{3}$. Thus, compared with their data, we concluded that the conduction processes in our samples are better interpreted in the context of the space-charge model.

\section{CONCLUSION}

We have investigated the temperature and frequency dependences of the dielectric permittivity and electric modulus in pure and $\mathrm{Ba}$-doped $\mathrm{Bi}_{2} \mathrm{Ti}_{4} \mathrm{O}_{11}$. We found that $\mathrm{Ba}$ doping decreased the antiferroelectric phase transition temperature due to reduction of the displacements of $\mathrm{Bi}^{3+}$ ions along the $b$ axis. Besides, we observed a dielectric dispersion in both samples and attributed it to the motion of the off-center Ti ions in the distorted oxygen octahedra. We also found a conduction process at high temperature, and suggested it to be associated with oscillations of space charge which is an accumulation of free charges at the two electrode interfaces.

\section{ACKNOWLEDGMENTS}

This work was supported by the Nebraska Research Initiative, the Nebraska-EPSCoR-NSF Grant No. EPS- 
9720643, and the U.S. Army Office under Grants No. DAAG 55-98-1-0273, No. DAAG 55-99-1-0106, and No. DAAD 19-02-1-0099.

${ }^{1}$ S. P. Yordanov, I. S. Ivanov, and Ch. P. Carapanov, J. Phys. D 31, 800 (1998).

${ }^{2}$ E. C. Subbarao, Phys. Rev. 122, 804 (1961).

${ }^{3}$ S. P. Yordanov, Ch. P. Carapanov, I. S. Ivanov, and P. T. Cholakov, Ferroelectrics 209, 541 (1998).

${ }^{4}$ E. C. Subbarao, J. Am. Ceram. Soc. 45, 564 (1962).

${ }^{5}$ V. Kahlenberg and H. Bohm, Acta Crystallogr., Sect. B: Struct. Sci. 51, 11 (1995).

${ }^{6}$ K. Hisano and K. Toda, Solid State Commun. 24, 247 (1977).

${ }^{7}$ H. S. Shulman, D. Damjamovic, and N. Setter, J. Am. Ceram. Soc. 83, 528 (2000).

${ }^{8}$ C. Jovalekic, M. Pavolvic, P. Osmokrovic, and M. Zdujic, IEEE Trans. Dielectr. Electr. Insul. 2, 409 (1995).

${ }^{9}$ L. Nistor, G. Van Tendeloo, S. Amelinckx, V. Kahlenberg, and H. Bohm, J. Solid State Chem. 119, 281 (1995).

${ }^{10}$ S. Havriliak and S. Negami, Polymer 8, 161 (1967).
${ }^{11}$ E. V. Colla, E. Yu Koroleva, N. M. Okuneva, and S. B. Vakhrushev, J. Phys.: Condens. Matter 4, 3671 (1992).

${ }^{12}$ K. Binder and A. P. Young, Rev. Mod. Phys. 58, 801 (1986).

${ }^{13}$ E. Courtens, Phys. Rev. B 33, 2975 (1986)

${ }^{14}$ M. Wubbenhorst and J. V. Turnhout, J. Non-Cryst. Solids 305, 40 (2002).

${ }^{15}$ P. B. Macedo, C. T. Moynihan, and R. Bose, Phys. Chem. Glasses 13, 171 (1972).

${ }^{16}$ C. A. Angell, Chem. Rev. 90, 523 (1990).

${ }^{17}$ I. M. Hodge, M. D. Ingram, and A. R. West, J. Electroanal. Chem. 74, 125 (1976).

${ }^{18}$ R. Gerhardt, J. Phys. Chem. Solids 55, 1491 (1994).

${ }^{19}$ J. S. Kim, J. Phys. Soc. Jpn. 70, 3129 (2001).

${ }^{20}$ G. I. Skanavi and E. N. Matveeva, Sov. Phys. JETP 3, 905 (1956).

${ }^{21}$ G. I. Skanavi, I. A. M. Ksendzov, V. A. Trigubenko, and V. G. Prokhvatilov, Sov. Phys. JETP 6, 250 (1958).

${ }^{22}$ D. W. Johnson, L. E. Cross, and F. A. Hummel, J. Appl. Phys. 41, 2828 (1970).

${ }^{23}$ R. Waser, T. Baiatu, and K. H. Hardtl, J. Am. Ceram. Soc. 73, 1645 (1990).

${ }^{24}$ R. Coelho, Rev. Phys. Appl. 18, 137 (1983).

${ }^{25}$ O. Bidault, P. Goux, M. Kchikech, M. Belkaoumi, and M. Maglione, Phys. Rev. B 49, 7868 (1994). 